http://jmscr.igmpublication.org/home/

ISSN (e)-2347-176x ISSN (p) 2455-0450

crossref DOI: https://dx.doi.org/10.18535/jmscr/v7i7.81

\title{
Segmental Follicular Vitiligo: A New Subtype
}

\author{
Authors \\ Mudita Gupta ${ }^{1}$, Saru Thakur ${ }^{2 *}$, Kuldeep Verma ${ }^{3}$, Reena Kumari Sharma ${ }^{4}$ \\ ${ }^{1}$ Assistant Professor, ${ }^{2,3}$ Post graduate student, ${ }^{4}$ Senior Resident, Deptt. of Dermatology, Leprosy and \\ Venereology, Indira Gandhi Medical College, Shimla, Himachal Pradesh \\ *Corresponding Author \\ Saru Thakur \\ Deptt. of Dermatology, Leprosy and Venereology, Indira Gandhi Medical College, Shimla, \\ Himachal Pradesh, India
}

\begin{abstract}
Vitiligo is a common disorder of pigmentation characterised by depigmented macules and patches. Autoimmune damage to the basal melanocytes leads to such presentation. Sometimes, there is involvement of melanocytes of hair matrixor outer root sheath, leading to depigmented hair or follicular vitiligo. It is a newer variant of vitiligo and has been reported uncommonly. We report a case of follicular vitiligo involving the beard area with no vitiligo lesions at any other body site and that too in a segmental distribution.

Keywords: follicular, segmental, vitiligo.
\end{abstract}

\section{Introduction}

Vitiligo is a common auto immune disorder characterised by the presence of depigmented macules and patches due to damage to the basal melanocytes. The pigment derived from the melanocytes and melanocytes stem cells of the hair follicles compensates the pigment loss in the vitiligo patches. But when these follicular melanocytes are themselves damaged, it causes follicular vitiligo ${ }^{(1)}$. This presence of depigmented hair in a patch of vitiligo, also called as leukotrichia, is considered a bad prognostic sign. Very few authors ${ }^{(2,3)}$ have reported follicular vitiligo and most of them either preceded or had vitiligo lesions elsewhere in the skin. In the present case, there was depigmented hair with no depigmented lesions elsewhere in the body and characteristically present in a segmental distribution. This is the first case of segmental follicular vitiligo to the best of our knowledge.

\section{Case Report}

A thirty years old male presented to dermatology OPD for assessment of white hair involving the beard region unilaterally on the right side. These were noticed by the patient since past one year and were gradually extending to the neck, maintaining the laterality of lesions. On examination, there was leukotrichia on right side of beard (figure 1,2) and chin region, while the interfollicular epidermis was spared. There were no other lesions over both the hairy and non-hairy parts of the skin. The patient denied any personal or family history of premature greying and 
vitiligo. Dermoscopy confirmed the absence of affection of the interfollicular epidermis with presence of depigmented hair.

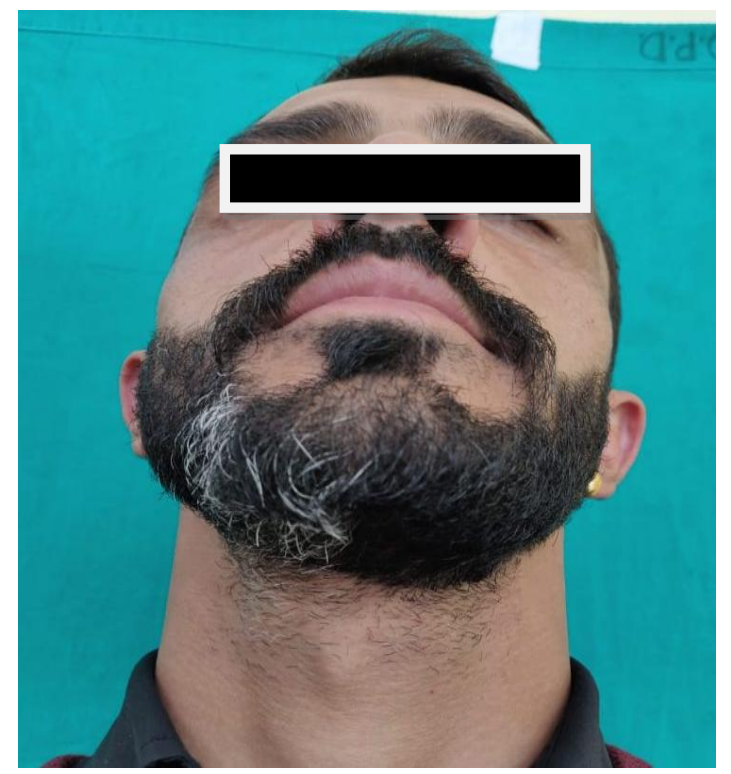

Figure 1: depigmented hair seen in the beard on right side.

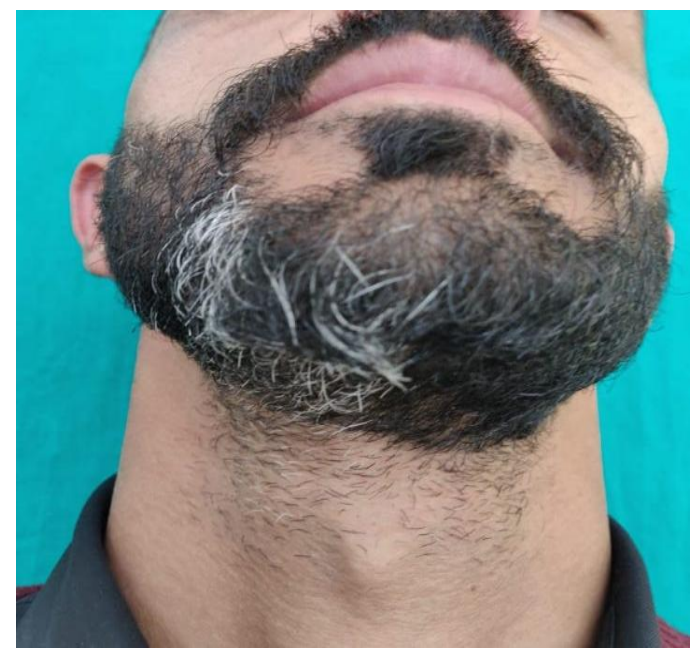

Figure 2: follicular vitiligo seen in a segmental distribution

\section{Discussion}

Follicular vitiligo is a recently proposed subtype of vitiligo, which primarily involves the melanocyte reservoir in the hair follicles ${ }^{(4)}$ with limited interfollicular compartment involvement. This entity was first described by Ezzedine $e t a l .^{(2)}$ in 2012 in a young black boy in whom leukotrichia preceded the appearance of depigmented macules. Gan et al. ${ }^{(3)}$ further reported a retrospective study of eight cases of follicular vitiligo with leucotrichia both in depigmented vitiligo macules and in normal appearing skin. Kamat et $a l^{(5)}$ reported generalized follicular vitiligo in a young boy. All these patients had cutaneous depigmentation also. Cabrera et al. ${ }^{(6)}$ reported eyelash follicular vitiligo and confirmed it dermatoscopically. There was absence of vitiligo elsewhere and in between the hair follicles as seen in our case.

Leukotrichia has been extensively reported invitiligo lesions in upto $45 \%$ of patients ${ }^{(6)}$. It is suggested that leukotrichia occurs as a secondary involvement of the hair, usually vellus hair, following a primary skin involvement. Follicular vitiligo on the contrary, is suggested to be a primary involvement of hair follicles and that too mostly terminal hair ${ }^{(6)}$. Hence, leukotrichia may be differentiated from follicular vitiligo.

In an attempt to systematize follicular vitiligo, it has been classified as following: (a) Premature greyness of hair, (b) Follicular vitiligo associated with ordinary vitiligo, (c) Follicular vitiligo in segmental vitiligo and (d) Follicular vitiligo in the form of greyness of body hair ${ }^{(1)}$.In our patient, white hair were present unilaterally in a segmental fashion, without any interfollicular involvement or cutaneous lesions. Hence, it may be considered as a new variant of follicular vitiligo.

Recognition of segmental vitiligo can help to reassure the patient that it usually does not spread to other parts of the body, stabilizes early and responds very well to surgical methods with probably a lower risk of recurrence. Recognition of this type of vitiligo would clearly be important in prognostication and choice of treatment modality.

\section{Conclusion}

Leukotrichia has been commonly observed in segmental vitiligo, but follicular vitiligo occurring segmentally has not been reported. Segmental follicular vitiligo may be considered as anew subtype of follicular vitiligo. 


\section{Declaration of Patient Consent}

The authors certify that they have obtained all appropriate patient consent forms, in which, the patient has given his consent for his images and other clinical information to be reported in the journal. The patient understands that name and initials will not be published and due efforts will be made to conceal identity, but anonymity cannot be guaranteed.

Financial Support and Sponsorship: Nil.

\section{Conflicts of Interest: Nil.}

\section{References}

1. Sharquie KE, Noaimi AA. Follicular vitiligo: the present clinical status. Our Dermatol Online. 2016;7(2):176-178.

2. Ezzedine K, Amazan E, Séneschal J, Cario-André $\mathrm{M}$, Léauté-Labrèze $\mathrm{C}$, Vergier B, et al. Follicular vitiligo: A new form of vitiligo. Pigment Cell Melanoma Res. 2012;25:527-9.

3. Gan EY, Cario-André M, Pain C, Goussot JF, Taïeb A, Seneschal J, et al. Follicular vitiligo: A report of 8 cases. J Am AcadDermatol 2016;74:1178-84.

4. Gopinath H, Karthikeyan K, Valeti M. A Lagging Lock: Hair Cycle Abnormalities in Follicular Vitiligo? Int $\mathrm{J}$ Trichology. 2017;9:142-3.

5. Kamat D, Prabhakaran N, Malathi M. Follicular vitiligo: A rare presentation of vitiligo. Indian J DermatolVenereolLeprol 2019;85:414-5.

6. Cabrera R, Recule F, Hojman L, Larrondo J. Follicular vitiligo: dermatoscopic features of a new subtype of vitiligo. An Bras Dermatol. 2019;94(1):120-1. 